\title{
INFINITE DIMENSIONAL TILTING MODULES OVER FINITE DIMENSIONAL ALGEBRAS
}

\author{
$\varnothing$ YVIND SOLBERG
}

\section{INTRODUCTION}

The theory for tilting and cotilting modules has its roots in the representation theory of finite dimensional algebras (artin algebras) generalizing Morita equivalence and duality. First through reflection functors studied in [14] and a module theoretic interpretation of these in [8], tilting modules of projective dimension at most one got an axiomatic description in [16, 39]. Among others, $[6,15,57]$ developed this theory further. These concepts were generalized in $[38,50]$ to tilting modules of arbitrary finite projective dimension. In the seminal paper [9] tilting and cotilting modules were characterized by special subcategories of the category of finitely presented modules. This paper started among other things the close connections between tilting and cotilting theory and homological conjectures studied further in $[21,40,42]$.

Generalizations of tilting modules of projective dimension at most one to arbitrary associative rings have been considered in [4, 24, 30, 49]. As tilting and cotilting modules in this general setting is not necessarily linked by applying a duality, a parallel development of cotilting modules were pursued among others in $[22,23,25,26,27,28,29]$. Definitions of tilting and cotilting modules of higher were introduced in [3] and [58], where the definition introduced in [3] being the most widely used now.

As the theory of tilting and cotilting modules has its origin in the representation theory of finite dimensional algebras, the current research on tilting and cotilting modules over more general rings seems also to return to finiteness conditions by considering modules of finite, cofinite and countable type. In this paper we consider finite dimensional algebras or artin algebras $\Lambda$, and review some of the properties and the structure of all tilting and cotilting modules. Of particular interest is the information these can give on the representation theory of finitely presented modules over $\Lambda$.

Let $\Lambda$ be an artin algebra, and let $\operatorname{Mod} \Lambda$ denote the category of all left $\Lambda$ modules. Our aim is to show that generalizing the characterization of finitely presented tilting and cotilting modules over $\Lambda$ to arbitrary tilting and cotilting modules in $\operatorname{Mod} \Lambda$ gives many of the results obtained for these modules. After recalling definitions and some basic results in section 1 , the next section gives analogues in $\operatorname{Mod} \Lambda$ of all the known characterizations of finitely presented tilting and cotilting modules given in [9]. Section 3 is devoted to discussing the finitistic dimension conjectures. Here the little finitistic dimension of $\Lambda$, when finite, is shown to be obtained as the projective dimension of a tilting module in $\operatorname{Mod} \Lambda$, and we investigate

Date: May 6, 2004.

Research supported by the Research Council of Norway and NTNU. 
when the big finitistic dimension is given by the projective dimension (the injective dimension) of a tilting (cotilting) module. We proceed in the next section to show that all finitely presented partial tilting and cotilting modules, when viewed as partial tilting and cotilting modules in $\operatorname{Mod} \Lambda$ always have a complement, contrary to within finitely presented modules. Moreover, the classical completion result for all finitely presented partial tilting and cotilting modules of projective and injective dimension at most one, respectively, is generalized for cotilting modules and only partially generalized for tilting modules. We end by giving the classification of all cotilting modules over a tame hereditary algebra.

\section{Definitions AND PRELiminaries}

In this section we recall the definitions and the preliminary results that we shall use throughout the paper.

Let $\Lambda$ be a ring. Denote by $\bmod \Lambda$ the full subcategory of Mod $\Lambda$ consisting of all finitely presented $\Lambda$-modules. For a module $M$ in $\operatorname{Mod} \Lambda$ we define the full subcategory (i) add $M$ to be the direct summands of all finite coproducts of copies of $M$, (ii) Add $M$ to be the direct summands of arbitrary coproducts of copies of $M$, and (iii) Prod $M$ to be the direct summands of arbitrary products of copies of $M$. For $M$ in $\bmod \Lambda$ let $\operatorname{Sub}(M)$ be the full subcategory of $\bmod \Lambda$ consisting of all submodules of a finite coproducts of copies of $M$. For a subcategory $\mathcal{C}$ of $\operatorname{Mod} \Lambda$ the full subcategory $\left\{Y \in \operatorname{Mod} \Lambda \mid \operatorname{Ext}_{\Lambda}^{i}(\mathcal{C}, Y)=(0)\right.$ for all $\left.i>0\right\}$ is denoted by $\mathcal{C}^{\perp}$. Dually ${ }^{\perp} \mathcal{C}$ denotes the full subcategory of $\operatorname{Mod} \Lambda$ given by $\{X \in \operatorname{Mod} \Lambda \mid$ $\operatorname{Ext}_{\Lambda}^{i}(X, \mathcal{C})=(0)$ for all $\left.i>0\right\}$. The full subcategory of $\operatorname{Mod} \Lambda$ consisting of all modules $X$ with a finite resolution

$$
0 \rightarrow C_{n} \rightarrow C_{n-1} \rightarrow \cdots \rightarrow C_{1} \rightarrow C_{0} \rightarrow X \rightarrow 0
$$

for some $n \geqslant 0$ and with $C_{i}$ in $\mathcal{C}$ is denoted by $\hat{\mathcal{C}}$. If all modules in $\operatorname{Mod} \Lambda$ is in $\widehat{\mathcal{C}}$ with the length $n$ bounded by some number $N<\infty$, we say that the resolution $\operatorname{dimension} \operatorname{resdim}_{\mathcal{C}}(\operatorname{Mod} \Lambda)$ of $\operatorname{Mod} \Lambda$ is finite, and otherwise it is infinite. Dually the full subcategory of $\operatorname{Mod} \Lambda$ consisting of all modules $Y$ with a finite coresolution

$$
0 \rightarrow Y \rightarrow C^{0} \rightarrow C^{1} \rightarrow \cdots \rightarrow C^{n-1} \rightarrow C^{n} \rightarrow 0
$$

for some $n \geqslant 0$ and with $C^{i}$ in $\mathcal{C}$ for all $i$ is denoted by $\check{\mathcal{C}}$. The injective dimension and the projective dimension of a module $X$ are denoted by $\operatorname{id}_{\Lambda} X$ and $\operatorname{pd}_{\Lambda} X$ respectively.

Now we give the definitions of a tilting and a cotilting module over an arbitrary ring $\Lambda$ from [3]. Recall that a $\Lambda$-module $T$ is a tilting module if

(T1) $\operatorname{pd}_{\Lambda} T<\infty$;

(T2) $\operatorname{Ext}_{\Lambda}^{i}(T, \amalg T)=(0)$ for all $i>0$ and all coproducts $\amalg T$ of copies of $T$;

(T3) there exists a long exact sequence $0 \rightarrow \Lambda \rightarrow T^{0} \rightarrow T^{1} \rightarrow \cdots \rightarrow T^{n-1} \rightarrow$ $T^{n} \rightarrow 0$ with $T^{i}$ in Add $T$ for all $i=0,1, \ldots, n$.

A module $T$ is called a partial tilting module if $T$ satisfies the conditions (T1) and (T2). If $T$ is a partial tilting module, then a module $T^{\prime}$ such that $T \amalg T^{\prime}$ is a tilting module is called a complement of $T$. A $\Lambda$-module $T$ is a cotilting module if

(C1) $\operatorname{id}_{\Lambda} T<\infty$;

(C2) $\operatorname{Ext}_{\Lambda}^{i}\left(\prod T, T\right)=(0)$ for all $i>0$ and all products $\prod T$ of copies of $T$;

(C3) there exists an injective generator $I$ and a long exact sequence $0 \rightarrow T_{n} \rightarrow$ $\cdots \rightarrow T_{1} \rightarrow T_{0} \rightarrow I \rightarrow 0$ with $T_{i}$ in $\operatorname{Prod} T$ for all $i=0,1, \ldots, n$. 
A module $T$ is called a partial cotilting module if $T$ satisfies the conditions (C1) and (C2). Dually we also define complements of partial cotilting modules. Also recall that a module $T$ in $\bmod \Lambda$ is a tilting or a cotilting module if $\operatorname{Add} T$ and $\operatorname{Prod} T$ are replaced by add $T$ and arbitrary coproducts and products are replaced by finite coproducts in the above definitions. In particular, a tilting or cotilting module in $\bmod \Lambda$ is still a tilting or cotilting module in $\operatorname{Mod} \Lambda$, respectively.

We denote by $\mathcal{I}^{\alpha}(\operatorname{Mod} \Lambda)$ and $\mathcal{P}^{\alpha}(\operatorname{Mod} \Lambda)$ for $\alpha$ in $\mathbb{N} \cup\{\infty\}$ the full subcategories of $\operatorname{Mod} \Lambda$ consisting of all modules $X$ with $\operatorname{id}_{\Lambda} X<\alpha$ and all modules $Y$ with $\operatorname{pd}_{\Lambda} Y<\alpha$, respectively. The categories $\mathcal{I}^{\alpha}(\operatorname{Mod} \Lambda)$ and $\mathcal{P}^{\alpha}(\operatorname{Mod} \Lambda)$ are examples of coresolving and resolving categories, that is, a subcategory $\mathcal{X}$ of $\operatorname{Mod} \Lambda$ is called resolving if $\mathcal{X}$ contains all projective modules and is closed under kernels of epimorphisms, direct summands and extensions. Coresolving is defined dually.

The big finitistic dimension Findim $\Lambda$ of $\Lambda$ is given by

$$
\sup \left\{\operatorname{pd}_{\Lambda} X \mid X \in \operatorname{Mod} \Lambda \text { with } \operatorname{pd}_{\Lambda} X<\infty\right\} .
$$

The little finitistic dimension findim $\Lambda$ of $\Lambda$ is defined as

$$
\sup \left\{\operatorname{pd}_{\Lambda} X \mid X \in \bmod \Lambda \text { with } \operatorname{pd}_{\Lambda} X<\infty\right\} .
$$

Let $\mathcal{X}$ be a class of $\Lambda$-modules. For a given $\Lambda$-module $C$, a map $\varphi: X \rightarrow C$ is a right $\mathcal{X}$-approximation of $C$ if $X$ is in $\mathcal{X}$ and $\operatorname{Hom}_{\Lambda}(\mathcal{X}, X) \stackrel{\operatorname{Hom}_{\Lambda}(\mathcal{X}, \varphi)}{\longrightarrow} \operatorname{Hom}_{\Lambda}(\mathcal{X}, C)$ is surjective. The approximation $\varphi$ is minimal if every endomorphism $f: X \rightarrow X$ with $\varphi f=\varphi$ is an isomorphism. If every $\Lambda$-module has a right $\mathcal{X}$-approximation, then $\mathcal{X}$ is called contravariantly finite in $\operatorname{Mod} \Lambda$.

One crucial result with respect to approximations is the Wakamatsu's Lemma [62]. It says the following.

Lemma 1.1. [9, Lemma 1.3] Let $\mathcal{X}$ be a class of $\Lambda$-modules closed under extensions, and let $0 \rightarrow Y \rightarrow X \stackrel{\varphi}{\rightarrow} C \rightarrow 0$ be an exact sequence of $\Lambda$-modules.

(a) If $\varphi$ is a minimal right $\mathcal{X}$-approximation, then $\operatorname{Ext}_{\Lambda}^{1}(\mathcal{X}, Y)=(0)$.

(b) If $\operatorname{Ext}_{\Lambda}^{1}(\mathcal{X}, Y)=(0)$ and $X$ is in $\mathcal{X}$, then $\varphi$ is a right $\mathcal{X}$-approximation.

If an right $\mathcal{X}$-approximation $\varphi: X \rightarrow C$ is surjective and $\operatorname{Ext}_{\Lambda}^{1}(\mathcal{X}, \operatorname{Ker} \varphi)=(0)$, the approximation is called special.

We leave it to the reader to define the dual concepts of all the above notions for approximations in $\operatorname{Mod} \Lambda$ and the corresponding notion in $\bmod \Lambda$.

\section{The SUBCATEGORY CORRESPONDENCE}

Let $\Lambda$ be an artin algebra. In Theorem 5.5 of the paper [9] tilting and cotilting modules in $\bmod \Lambda$ were characterized by certain subcategories of $\bmod \Lambda$. This characterization has lead to many fruitful applications as reviewed in [51]. So it is natural to ask if there is a similar correspondence for arbitrary tilting and cotilting modules over $\Lambda$. This section is devoted to discussing such correspondences. For a closely related investigation of the associated cotorsion pairs see [59].

First we recall the characterization of equivalence classes of tilting and cotilting modules in $\bmod \Lambda$ given in [9]. Here we say that two tilting or cotilting modules $T$ and $T^{\prime}$ in $\bmod \Lambda$ are equivalent if add $T=\operatorname{add} T^{\prime}$. Note that in the following result all modules and subcategories are in $\bmod \Lambda$.

Theorem 2.1 ([9, Theorem 5.5]). Let $T$ be a selforthogonal module. 
(a) $T \mapsto{ }^{\perp} T$ gives a one-to-one correspondence between equivalence classes of cotilting modules and contravariantly finite resolving subcategories $\mathcal{X}$ of $\bmod \Lambda$ with $\hat{\mathcal{X}}=\bmod \Lambda$. The inverse is given by $\mathcal{X} \mapsto \mathcal{X} \cap \mathcal{X}^{\perp}$.

(b) $T \mapsto \widehat{\operatorname{add} T}$ gives a one-to-one correspondence between equivalence classes of cotilting modules and covariantly finite coresolving subcategories $\mathcal{Y}$ of $\mathcal{I}^{\infty}(\bmod \Lambda)$. The inverse is given by $\mathcal{Y} \mapsto{ }^{\perp} \mathcal{Y} \cap \mathcal{Y}$.

(c) $T \mapsto T^{\perp}$ gives a one-to-one correspondence between equivalence classes of tilting modules and covariantly finite coresolving subcategories $\mathcal{Y}$ of with $\check{\mathcal{Y}}=\bmod \Lambda$. The inverse is given by $\mathcal{Y} \mapsto^{\perp} \mathcal{Y} \cap \mathcal{Y}$.

(d) $T \mapsto \overline{\operatorname{add} T}$ gives a one-to-one correspondence between equivalence classes of tilting modules and contravariantly finite resolving subcategories $\mathcal{X}$ of $\mathcal{P}^{\infty}(\bmod \Lambda)$. The inverse is given by $\mathcal{X} \mapsto \mathcal{X} \cap \mathcal{X}^{\perp}$.

The usual duality $D: \bmod \Lambda \rightarrow \bmod \Lambda^{\mathrm{op}}$ maps tilting modules to cotilting modules and vice versa. So a result for one case automatically yields a result for the other. Given this remark it is superfluous to give the correspondences both for tilting and cotilting modules. However since a result for tilting modules in Mod $\Lambda$ can not be translated to a result about cotilting modules by applying the duality $D$, we have chosen to give all correspondences in order to clearly see the relationship with the analogous correspondences in $\operatorname{Mod} \Lambda$.

We want to present analogues of the above characterizations for tilting and cotilting modules in $\operatorname{Mod} \Lambda$. As infinitely generated tilting and cotilting modules $T$ are defined in terms Add $T$ and Prod $T$ the following are natural definitions. Two tilting (or cotilting) modules $T$ and $T^{\prime}$ in $\operatorname{Mod} \Lambda$ are equivalent if $\operatorname{Add} T=\operatorname{Add} T^{\prime}$ (or $\left.\operatorname{Prod} T=\operatorname{Prod} T^{\prime}\right)$.

We first discuss the situation for cotilting modules over $\Lambda$. A first approximation to such a characterization was found in [3], and it reads as follows (this result is true for any ring).

Theorem 2.2 ([3, Theorem 4.2]). Let $\mathcal{X}$ be class of modules in $\operatorname{Mod} \Lambda$ closed under kernels of epimorphisms and such that $\mathcal{X} \cap \mathcal{X}^{\perp}$ is closed under products. The following are equivalent.

(a) There exists a cotilting module $T$ with $\operatorname{id}_{\Lambda} T \leqslant n$ such that $\mathcal{X}={ }^{\perp} T$;

(b) Every left $\Lambda$-module has a special $\mathcal{X}$-approximation and all modules $Y$ in $\mathcal{X}^{\perp}$ have $\operatorname{id}_{\Lambda} Y \leqslant n$.

From our view point and for our purposes this is not a true generalization of the characterization given of tilting and cotilting modules in [9], since it involve both a category and its Ext-orthogonal category. A characterization of the equivalence classes of cotilting modules in terms of subcategories of $\operatorname{Mod} \Lambda$ is given as follows.

Theorem 2.3 ([47, Theorem 5.6]). Let $T$ be a selforthogonal module in Mod $\Lambda$. The map $T \mapsto{ }^{\perp} T$ gives a one-to-one correspondence between equivalence classes of cotilting modules over $\Lambda$ and resolving subcategories $\mathcal{X}$ of $\operatorname{Mod} \Lambda$ closed under products with $\hat{\mathcal{X}}=\operatorname{Mod} \Lambda$, such that every $\Lambda$-module has a special right $\mathcal{X}$ approximation. The inverse is given by $\mathcal{X} \mapsto \mathcal{X} \cap \mathcal{X}^{\perp}$.

Remark. This result is in fact true for any $\operatorname{ring} R$ by replacing $\widehat{\mathcal{X}}=\operatorname{Mod} R$ by $\operatorname{resdim}_{\mathcal{X}}(\operatorname{Mod} R)<\infty$ as formulated in [47]. So we see that the only real difference with Theorem 2.1 (a) is that the corresponding category should be closed under products, since in $\bmod \Lambda$ any module having a right $\mathcal{X}$-approximation with $\mathcal{X}$ in 
$\bmod \Lambda$ has a minimal right approximation. In addition, when the category $\mathcal{X}$ is extension closed, then by Wakamatsu's Lemma a minimal approximation is special.

It is natural to ask if all the known characterizations of tilting and cotilting modules in $\bmod \Lambda$ have counterparts in $\operatorname{Mod} \Lambda$. We first consider an analogue of Theorem 2.1 (b).

Proposition 2.4. Let $T$ be a selforthogonal module in $\operatorname{Mod} \Lambda$. The map $T \mapsto$ $\widehat{\operatorname{Prod} T}$ gives a one-to-one correspondence between equivalence classes of cotilting modules over $\Lambda$ and coresolving subcategories $\mathcal{Y}$ of $\operatorname{Mod} \Lambda$

(i) closed under products,

(ii) contained in $\mathcal{I}^{\infty}(\operatorname{Mod} \Lambda)$,

(iii) every $\Lambda$-module has a special left $\mathcal{Y}$-approximation,

(iv) if $0 \rightarrow \Lambda / \mathfrak{r} \rightarrow Y^{\Lambda / \mathfrak{r}} \rightarrow X^{\Lambda / \mathfrak{r}} \rightarrow 0$ is a special left $\mathcal{Y}$-approximation, then the category $\left\{X \in \operatorname{Mod} \Lambda \mid \operatorname{Ext}_{\Lambda}^{1}\left(X, Y^{\Lambda / \mathfrak{r}}\right)=(0)\right\}$ is closed under products.

Proof. Assume first that $T$ is a cotilting module in $\operatorname{Mod} \Lambda$. We claim that $\widehat{\operatorname{Prod} T}=$ $\left({ }^{\perp} T\right)^{\perp}$. Using that Prod $T$ is contained in $\left({ }^{\perp} T\right)^{\perp}$, we conclude that $\left({ }^{\perp} T\right)^{\perp}$ contains $\widehat{\operatorname{Prod} T}$. Taking a sequence $\cdots \rightarrow X_{2} \stackrel{f_{2}}{\longrightarrow} X_{1} \stackrel{f_{1}}{\longrightarrow} X_{0} \stackrel{f_{0}}{\longrightarrow} Z \rightarrow 0$ of special right ${ }^{\perp} T$ approximations of a module $Z$ in $\left({ }^{\perp} T\right)^{\perp}$, the kernels $\operatorname{Ker} f_{i}$ are in $\left({ }^{\perp} T\right)^{\perp}$ and $X_{i}$ are in $\operatorname{Prod} T$. Since $\left({ }^{\perp} T\right)^{\perp}$ is contained in $\mathcal{I}^{n+1}(\operatorname{Mod} \Lambda)$ for some $n<\infty$, the module $\operatorname{Ker} f_{n-1}$ is in $\operatorname{Prod} T$. Hence $Z$ is in $\widehat{\operatorname{Prod} T}$ and $\left({ }^{\perp} T\right)^{\perp}=\widehat{\operatorname{Prod} T}$, which clearly is closed under products. Then by [9, Proposition 1.8] the subcategory $\widehat{\operatorname{Prod} T}$ is coresolving where all modules have a special left $\widehat{\operatorname{Prod} T}$-approximation.

Dual to Theorem 3.1 in [47] any module in $\widehat{\operatorname{Prod} T}$ is a direct factor of a module having a finite filtration in products of copies of $Y^{\Lambda / \mathfrak{r}}$. Then it is easy to see that $\left\{X \in \operatorname{Mod} \Lambda \mid \operatorname{Ext}_{\Lambda}^{1}\left(X, Y^{\Lambda / \mathfrak{r}}\right)=(0)\right\}={ }^{\perp} T$ and therefore closed under products.

Conversely, let $\mathcal{Y}$ be a subcategory of $\operatorname{Mod} \Lambda$ satisfying the properties (i)-(iv). Then ${ }^{\perp} \mathcal{Y}$ is resolving and all modules have a special right ${ }^{\perp} \mathcal{Y}$-approximation. Dual to as in the proof of Theorem 3.1 in [47] a module is in $\mathcal{Y}$ if and only if it is a direct factor of a module having a finite filtration in products of copies of $Y^{\Lambda / \mathrm{r}}$. Hence $\mathcal{Y}$ is contained in $\mathcal{I}^{n+1}(\operatorname{Mod} \Lambda)$ for some $n<\infty$. Then we infer that $\{X \in$ $\left.\operatorname{Mod} \Lambda \mid \operatorname{Ext}_{\Lambda}^{1}\left(X, Y^{\Lambda / \mathfrak{r}}\right)=(0)\right\}={ }^{\perp} \mathcal{Y}$ and that $\widehat{{ }^{\mathcal{Y}}}=\operatorname{Mod} \Lambda$. Then ${ }^{\perp} \mathcal{Y}={ }^{\perp} T$ for some cotilting module $T$ with $\operatorname{Prod} T={ }^{\perp} \mathcal{Y} \cap \mathcal{Y}$. Furthermore by the above we have that $\mathcal{Y}=\widehat{\operatorname{Prod} T}$.

Now we give the analogues of (c) and (d) in Theorem 2.1. The proofs are dual to those of Theorem 2.3 and Proposition 2.4.

Proposition 2.5. Let $T$ be a selforthogonal module in $\operatorname{Mod} \Lambda$.

(a) The map $T \mapsto T^{\perp}$ gives a one-to-one correspondence between equivalence classes of tilting modules over $\Lambda$ and coresolving subcategories $\mathcal{Y}$ of $\operatorname{Mod} \Lambda$ closed under coproducts with $\breve{\mathcal{Y}}=\operatorname{Mod} \Lambda$, such that every $\Lambda$-module has a special left $\mathcal{Y}$-approximation.

(b) The map $T \mapsto \overline{\operatorname{Add} T}$ gives a one-to-one correspondence between equivalence classes of tilting modules over $\Lambda$ and resolving subcategories $\mathcal{X}$ of $\operatorname{Mod} \Lambda$

(i) closed under coproducts,

(ii) contained in $\mathcal{P}^{\infty}(\operatorname{Mod} \Lambda)$,

(iii) every $\Lambda$-module has a special right $\mathcal{X}$-approximation, 
(iv) if $0 \rightarrow Y_{\Lambda / \mathfrak{r}} \rightarrow X_{\Lambda / \mathfrak{r}} \rightarrow \Lambda / \mathfrak{r} \rightarrow 0$ is a special right $\mathcal{X}$-approximation, then the category $\left\{Y \in \operatorname{Mod} \Lambda \mid \operatorname{Ext}_{\Lambda}^{1}\left(X_{\Lambda / \mathfrak{r}}, Y\right)=(0)\right\}$ is closed under coproducts.

In [9] the subcategory ${ }^{\perp} T$ of $\bmod \Lambda$ is shown not only to be contravariantly finite for a cotilting module $T$ in $\bmod \Lambda$, but also to be covariantly finite (see [9, Corollary 5.10]). Using subcategories of $\operatorname{Mod} \Lambda$ a resolving and contravariantly finite subcategory of $\bmod \Lambda$ is shown also to be covariantly finite in [46, Corollary 2.6]. Is something similar true for subcategories of $\operatorname{Mod} \Lambda$ ?

We end the discussion on arbitrary tilting and cotilting modules by considering the pure-injectivity of cotilting modules. Recall that a module $M$ is pure-injective if the natural map $M \rightarrow D^{2}(M)$ splits [45]. It is conjectured that all cotilting modules are pure-injective. This is proven for cotilting modules of injective dimension at most one in [11]. The general case is open. However, there exist characterizations of when a cotilting module is pure-injective. These characterizations can be found in [12] and [47, Theorem 5.7] (true for any ring).

Theorem 2.6. Let $T$ be a cotilting $\Lambda$-module. Then the following are equivalent.

(a) ${ }^{\perp} T$ is closed under pure factor modules,

(b) ${ }^{\perp} T$ is closed under pure submodules,

(c) ${ }^{\perp} T$ is closed under filtered colimits,

(d) every $\Lambda$-module has a minimal right ${ }^{\perp} T$-approximation and $D^{2}\left({ }^{\perp} T\right) \subseteq{ }^{\perp} T$,

(e) $T$ is pure-injective.

As already indicated by the above the theory for tilting and cotilting modules is in particular rich both for modules in $\bmod \Lambda$ and $\operatorname{Mod} \Lambda$ when restricted to projective and injective dimension at most one, respectively. In particular for $\bmod \Lambda$ every partial tilting module of projective dimension at most one can be completed to a tilting module (see [15]). This question we address in section 4 for tilting and cotilting modules in $\operatorname{Mod} \Lambda$. Furthermore, all torsion pairs $(\mathcal{T}, \mathcal{F})$ in $\bmod \Lambda$ with $\mathcal{F}=\operatorname{Sub}(M)$ for some $M$ in $\bmod \Lambda$ and with $\Lambda$ in $\mathcal{F}$ are in one-to-one correspondence with cotilting modules $T$ of injective dimension at most one [10] $(\mathcal{F}=\operatorname{Sub}(T))$. However there are torsion pairs in $\bmod \Lambda$ not induced by a cotilting (or tilting) module in $\bmod \Lambda$. For example, for the Kronecker algebra let $\mathcal{F}$ be the additive closure of the preprojective modules and any proper non-empty set of tubes in the Auslander-Reiten quiver. But in [17] it is shown that all torsion pairs $(\mathcal{T}, \mathcal{F})$ with $\Lambda$ in $\mathcal{F}$ are induced by cotilting modules of injective dimension at most one in $\operatorname{Mod} \Lambda$. We end this section by explaining this correspondence (true for left noetherian rings). For a subcategory $\mathcal{C}$ in $\bmod \Lambda$ denote by $\lim \mathcal{C}$ the full additive subcategory of all direct summands of the filtered colimits of modules in $\mathcal{C}$.

Theorem 2.7. There is a bijection between torsion pairs $(\mathcal{T}, \mathcal{F})$ in $\bmod \Lambda$ with $\Lambda$ in $\mathcal{F}$ and equivalence classes of cotilting modules of injective dimension at most one in $\operatorname{Mod} \Lambda$.

A cotilting module $T$ of injective dimension at most one corresponding to a torsion pair $(\mathcal{T}, \mathcal{F})$ satisfies $\operatorname{Prod} T=\lim _{\longrightarrow} \mathcal{F} \cap\left(\underline{\lim }_{\longrightarrow} \mathcal{F}\right)^{\perp}$ and ${ }^{\perp} T \cap \bmod \Lambda=\mathcal{F}$.

Proof. We sketch the proof of one direction to illustrate the use of the results in this section. Let $(\mathcal{T}, \mathcal{F})$ be a torsion pair in $\bmod \Lambda$ with $\Lambda$ in $\mathcal{F}$. By [31] $\left(\stackrel{\lim }{\mathcal{T}}, \lim _{\longrightarrow} \mathcal{F}\right)$ is a torsion pair in $\operatorname{Mod} \Lambda$. Note that any torsion free class of a torsion pair in $\operatorname{Mod} \Lambda$ containing $\Lambda$ is a resolving subcategory. Hence $\lim _{\longrightarrow} \mathcal{F}$ is resolving and closed 
under products. Since a first syzygy of any module in $\operatorname{Mod} \Lambda$ is in $\underset{\lim }{\mathcal{F}}$, we have that $\overleftrightarrow{\lim \mathcal{F}}=\operatorname{Mod} \Lambda$ and $\left(\lim _{\longrightarrow} \mathcal{F}\right)^{\perp}$ is contained in $\mathcal{I}^{2}(\operatorname{Mod} \Lambda)$. Every $\Lambda$-module has a minimal (special) right $\underset{\lim }{\longrightarrow} \mathcal{F}$-approximation by [17, Lemma 1.3] or [47, Theorem 2.6], since $\lim _{\longrightarrow} \mathcal{F}$ is resolving and closed under products and filtered colimits. Hence by Theorem 2.3 and 2.6 we have $\lim _{\longrightarrow} \mathcal{F} \cap(\underline{\lim } \mathcal{F})^{\perp}=\operatorname{Prod} T$ for some pure-injective cotilting module of injective dimension at $\overrightarrow{m o s t}$ one in $\operatorname{Mod} \Lambda$.

A different characterization of these torsion pairs when the ring is left or right artinian can be found in [59, Theorem 3.7]. For related information and examples over concealed canonical algebras see [52].

\section{The FINITISTIC DIMENSION CONJECTURES}

The main interest in this section is the finitistic conjectures for an artin algebra, that is, when is Findim $\Lambda=\operatorname{findim} \Lambda$ and when is findim $\Lambda$ finite. The first of these conjectures was disproved by in [41], and the second one has been proven for

(i) monomial algebras in [36] (again in [44]),

(ii) radical cube zero (and even more generally for algebras with Loewy length $2 n+1$ and $\Lambda / \mathfrak{r}^{n}$ of finite representation type, see [32]) in [37] ([43]),

(iii) when $\mathcal{P}^{\infty}(\bmod \Lambda)$ is contravariantly finite in [9],

(iv) when the representation dimension of $\Lambda$ is at most 3 in [43] (all special biserial algebras has representation dimension at most 3 , see [33]).

None of the above proofs of the finiteness of findim $\Lambda$ directly involve a tilting or a cotilting module. The results on relations between these conjectures and tilting and cotilting modules are more in the direction of finding test classes of modules for these conjectures, and if the dimensions are finite to show that they are obtained as the projective or the injective dimension of a tilting or cotilting module, respectively.

First we discuss how findim $\Lambda$ is obtained as the projetive dimension of a tilting module in $\operatorname{Mod} \Lambda$ when it is finite.

Proposition 3.1 ([5, Proposition 2.3]). For any positive integer $n$ there is a tilting module $T_{n}$ with projective dimension at most $n$ and $T^{\perp}=\mathcal{P}^{n+1}(\bmod \Lambda)^{\perp}$. If findim $\Lambda \geqslant n$, then $T_{n}$ has projective dimension $n$.

Proof. The category $\mathcal{Y}=\mathcal{P}^{n+1}(\bmod \Lambda)^{\perp}$ is clearly coresolving. Since $\mathcal{P}^{n+1}(\bmod \Lambda)$ consists only of finitely presented modules, $\mathcal{Y}$ is closed under coproducts. Every $\Lambda$-module has a special left $\mathcal{Y}^{\perp}$-approximation by [35, Theorem 10]. It is also clear that $\check{\mathcal{Y}}=\operatorname{Mod} \Lambda$. By Proposition 2.5 there exists a tilting module $T_{n}$ such that $T_{n}^{\perp}=\mathcal{Y}$. The last claim is left to the reader.

This immediately gives the following relationship between the finitistic dimensions Findim $\Lambda$ and findim $\Lambda$ (for the second statement see [19, Corollary 2.2]).

Corollary 3.2. (a) Findim $\Lambda \geqslant \sup \left\{\operatorname{pd}_{\Lambda} T \mid T\right.$ tilting module $\} \geqslant$ findim $\Lambda$.

(b) Findim $\Lambda \geqslant \sup \left\{\operatorname{id}_{\Lambda^{\mathrm{op}}} T \mid T\right.$ pure-injective cotilting $\Lambda^{\mathrm{op}}$-module $\} \geqslant$ findim $\Lambda$.

In view of this result an obvious question is: when Findim $\Lambda$ is finite or findim $\Lambda$ is finite, does there exist tilting or cotilting modules with projective or injective dimensions equal to one of the finitistic dimensions? It seems to be unknown in general if Findim $\Lambda$ always can be obtained in this way when it is finite. But we give some sufficient conditions later. 
It was first proved in $[9]$ that if $\mathcal{P}^{\infty}(\bmod \Lambda)$ is contravariantly finite, then findim $\Lambda$ is finite. It is easy to show using Theorem 2.1 (d) that then findim $\Lambda$ is obtained as the projective dimension of a finitely presented tilting module.

We show next that the finitistic dimension findim $\Lambda$ always is obtained by the projective dimension of a tilting module when it is finite.

Theorem 3.3. The following are equivalent.

(a) findim $\Lambda<\infty$.

(b) there exists a tilting module $T$ such that $T^{\perp}=\mathcal{P}^{\infty}(\bmod \Lambda)^{\perp}$.

(c) $\mathcal{P}^{\infty} \overline{(\bmod \Lambda)^{\perp}}=\operatorname{Mod} \Lambda$.

If any of these are true, then findim $\Lambda=\operatorname{pd}_{\Lambda} T$.

The equivalence of (a) and (b) is given for left noetherian rings in [5, Theorem 2.6]. The equivalence of (b) and (c) is an immediate consequence of Proposition 2.5 noting that $\mathcal{P}^{\infty}(\bmod \Lambda)^{\perp}$ always is coresolving and closed under coproducts, and every $\Lambda$-module has a special left $\mathcal{P}^{\infty}(\bmod \Lambda)^{\perp}$-approximation.

As a consequence of the above result Findim $\Lambda$ is obtained as the projective dimension of a tilting module $T$ whenever Findim $\Lambda$ and findim $\Lambda$ are equal. When $\mathcal{P}^{\infty}(\bmod \Lambda)$ is contravariantly finite in $\bmod \Lambda$ then Findim $\Lambda$ and findim $\Lambda$ are shown to be equal in [42] (for an alternative proof see [5, Corollary 4.3]). Hence this gives a situation where also Findim $\Lambda$ is realized as the projective dimension of a tilting module. Next we give a new criterion for when Findim $\Lambda$ is obtained as the projective dimension of a tilting module.

Proposition 3.4. $\quad$ (a) Assume that Findim $\Lambda<\infty$. The category $\mathcal{P}^{\infty}(\operatorname{Mod} \Lambda)^{\perp}$ is closed under coproducts if and only if there exists a tilting module $T$ such that $\overline{\operatorname{Add} T}=\mathcal{P}^{\infty}(\operatorname{Mod} \Lambda)$. In this case Findim $\Lambda=\operatorname{pd}_{\Lambda} T$.

(b) Assume that Findim $\Lambda^{\mathrm{op}}<\infty$. The category ${ }^{\perp} \mathcal{I}^{\infty}(\operatorname{Mod} \Lambda)$ is closed under products if and only if there exists a cotilting module $T$ such that $\widehat{\operatorname{Prod} T}=$ $\mathcal{I}^{\infty}(\operatorname{Mod} \Lambda)$. In this case Findim $\Lambda^{\mathrm{op}}=\operatorname{id}_{\Lambda} T$.

When Findim $\Lambda=n$ is finite, then the subcategory $\mathcal{P}^{\infty}(\operatorname{Mod} \Lambda)$ is equal to $\{X \in$ $\left.\operatorname{Mod} \Lambda \mid \operatorname{Ext}_{\Lambda}^{1}\left(X, \Omega_{\Lambda}^{-n}(\Lambda / \mathfrak{r})\right)=(0)\right\}$. This subcategory is resolving and every $\Lambda$ module has a minimal (special) right $\mathcal{P}^{\infty}(\operatorname{Mod} \Lambda)$-approximation by [34, Corollary 10]. The condition (iv) in Proposition 2.5 amounts to saying that $\mathcal{P}^{\infty}(\operatorname{Mod} \Lambda)^{\perp}$ is closed under coproducts. Similar arguments are used in (b), hence the above result is then a direct consequence of Proposition 2.4 and Proposition 2.5. For related results see also [5, Section 3].

The categories $\mathcal{P}^{n}(\operatorname{Mod} \Lambda)$ are proven to be contravariantly finite for any $n \geqslant 1$ in [1]. Then using similar arguments as in [9, Corollary 3.10] one can show that the following are equivalent (i) Findim $\Lambda$ is finite, (ii) $\mathcal{P}^{\infty}(\operatorname{Mod} \Lambda)$ is contravariantly finite and (iii) $\mathcal{P}^{\infty}(\operatorname{Mod} \Lambda$ ) is closed under coproducts (see [47, Corollary 2.7]).

We end this section by characterizing the cotilting module in Proposition 3.4 (b). To this end we use the characterization of cotilting modules to define further structures on the class of cotilting modules.

Let Cotilt $\Lambda$ denote the set of all equivalence classes of pure-injective cotilting modules in Mod $\Lambda$ (shown to be a set for any ring in [19]). We can partially order the cotilting modules according to the Hasse diagram of the subcategories ${ }^{\perp} T$ for $T$ in Cotilt $\Lambda$. Such a Hasse diagram of the subcategories ${ }^{\perp} T$ in $\bmod \Lambda$ for the set of all (co)tilting modules $T$ in $\bmod \Lambda$ has been considered in various contexts in 
$[54,60]$ (also see [61]). The Hasse diagram of Cotilt $\Lambda$ was considered in [19, 20], and it was shown to be a lattice in [19].

The characterization of cotilting modules in Theorem 2.3 imply that for any subset $\left\{T_{i}\right\}_{i \in I}$ of Cotilt $\Lambda$ with $\sup \left\{\operatorname{id}_{\Lambda} T_{i}\right\}_{i \in I}<\infty$, there exists a cotilting module $T$ in Cotilt $\Lambda$ such that ${ }^{\perp} T=\bigcap_{i \in I}{ }^{\perp} T_{i}$. Moreover, $\operatorname{id}_{\Lambda} T=\sup \left\{\operatorname{id}_{\Lambda} T_{i}\right\}_{i \in I}$. In particular, when Findim $\Lambda^{\mathrm{op}}$ is finite there is a unique minimal cotilting module $T_{\min }$ in Cotilt $\Lambda$ with $\operatorname{id}_{\Lambda} T=\sup \left\{\operatorname{id}_{\Lambda} T\right\}_{T \in \operatorname{Cotilt} \Lambda}$. It is unknown whether or not Findim $\Lambda^{\mathrm{op}}=\operatorname{id}_{\Lambda} T_{\min }$. We want to compare $T_{\min }$ to the module defined in the following lemma (see [19, Lemma 3.1 and 3.2]).

Lemma 3.5. Suppose that Findim $\Lambda^{\mathrm{op}}$ is finite.

(a) There exists a $\Lambda$-module $T$ such that

$$
{ }^{\perp} \mathcal{I}^{\infty}(\operatorname{Mod} \Lambda) \cap \mathcal{I}^{\infty}(\operatorname{Mod} \Lambda)=\operatorname{Add} T .
$$

(b) $\mathcal{I}^{\infty}(\operatorname{Mod} \Lambda)=\widehat{\operatorname{Add} T}$, where all the resolutions in $\operatorname{Add} T$ can be chosen to have length at most Findim $\Lambda^{\mathrm{op}}$.

(c) $\operatorname{id}_{\Lambda} T=$ Findim $\Lambda^{\mathrm{op}}$.

Denote the module $T$ in the above lemma by $T_{\mathrm{inj}}$. When Findim $\Lambda^{\mathrm{op}}$ is finite it is immediate that (i) $\operatorname{id}_{\Lambda} T_{\mathrm{inj}}<\infty$, (ii) $\operatorname{Ext}_{\Lambda}^{i}\left(T_{\mathrm{inj}}, T_{\mathrm{inj}}\right)=(0)$ for all $i>0$ and (iii) there exists a long exact sequence

$$
0 \rightarrow T_{n} \rightarrow T_{n-1} \rightarrow \cdots \rightarrow T_{1} \rightarrow T_{0} \rightarrow D(\Lambda) \rightarrow 0
$$

with $T_{i}$ in Add $T_{\mathrm{inj}}$ for all $i$. So $T_{\mathrm{inj}}$ is very close to being a cotilting module, but nevertheless if Findim $\Lambda^{\mathrm{op}}$ is finite, then it is obtained on a selforthogonal module. Moreover, we have ${ }^{\perp} \operatorname{Add} T_{\text {inj }}={ }^{\perp} \mathcal{I}^{\infty}(\operatorname{Mod} \Lambda)$.

The following result points out a relationship between the modules $T_{\text {inj }}$ and $T_{\min }$ and the dimensions $\operatorname{id}_{\Lambda} T_{\mathrm{inj}}$ and $\operatorname{id}_{\Lambda} T_{\min }$ when Findim $\Lambda^{\mathrm{op}}$ is finite (see [19, Theorem 3.3]). Recall that a modules $M$ in $\operatorname{Mod} \Lambda$ is called product complete if Add $M=\operatorname{Prod} M$, and it is called $\Sigma$-pure-injective if every coproduct $M^{(\alpha)}$ is pure-injective.

Theorem 3.6. Suppose that Findim $\Lambda^{\mathrm{op}}$ is finite. Then the following are equivalent.

(a) ${ }^{\perp} \mathcal{I}^{\infty}(\operatorname{Mod} \Lambda)={ }^{\perp} T_{\min }$,

(b) ${ }^{\perp} \mathcal{I}^{\infty}(\operatorname{Mod} \Lambda)$ is closed under products,

(c) $T_{\text {inj }}$ is product complete,

(d) $T_{\mathrm{inj}}$ is a $\Sigma$-pure-injective cotilting module.

Moreover, when one of these conditions holds $T_{\min }$ and $T_{\mathrm{inj}}$ are equivalent cotilting modules and Findim $\Lambda^{\mathrm{op}}=\operatorname{id}_{\Lambda} T_{\min }$.

\section{Complements of tilting And cotilting modules}

As already mentioned, a classical result in the theory of tilting modules in $\bmod \Lambda$ says that for any partial tilting module $T$ of projective dimension at most one, there exists a module $T^{\prime}$ in $\bmod \Lambda$ such that $T \amalg T^{\prime}$ is a tilting module with $T^{\perp}=\left(T \amalg T^{\prime}\right)^{\perp}$ (the Bongartz construction or complement). For a partial tilting module $T$ of projective dimension at least two in $\bmod \Lambda$, such a complement $T^{\prime}$ in $\bmod \Lambda$ does not always exist as shown in [53]. This naturally gives rise to at least two questions: (1) Does the Bongartz construction of a complement generalize to partial tilting (or cotilting) modules of projective (injective) dimension at most one in $\operatorname{Mod} \Lambda$ ? 
(2) Does any partial tilting or cotilting module $T$ in $\bmod \Lambda$ have a complement when considered as a partial tilting or cotilting module in Mod $\Lambda$ ? This section is devoted to discussing these questions. The answer to the second question is always yes, while the first question is answered in full for cotilting modules and only partially for tilting modules.

First we discuss a criterion for existence of complements of partial tilting and cotilting modules. The following is an easy consequence of our characterizations of tilting and cotilting modules in terms of subcategories of $\operatorname{Mod} \Lambda$.

Proposition 4.1 ([47, Proposition 6.1]). $\quad$ (a) Let $T$ be a partial tilting module. Then $T$ has a complement if and only if $T^{\perp}$ contains a coresolving subcategory $\mathcal{Y}$ containing Add $T$ and closed under coproducts with $\breve{\mathcal{Y}}=\operatorname{Mod} \Lambda$, such that every $\Lambda$-module has a special left $\mathcal{Y}$-approximation.

(b) Let $T$ be a partial cotilting module. Then $T$ has a complement if and only if ${ }^{\perp} T$ contains a resolving subcategory $\mathcal{X}$ containing $\operatorname{Prod} T$ and closed under products with $\widehat{\mathcal{X}}=\operatorname{Mod} \Lambda$, such that every $\Lambda$-module has a special right $\mathcal{X}$-approximation.

Recall that for an extension closed subcategory $\mathcal{X}$ of $\operatorname{Mod} \Lambda$ a module $M$ in $\mathcal{X}$ is called Ext-injective in $\mathcal{X}$ if $\operatorname{Ext}_{\Lambda}^{1}(\mathcal{X}, M)=(0)$ and Ext-projective in $\mathcal{X}$ if $\operatorname{Ext}_{\Lambda}^{1}(M, \mathcal{X})=(0)$. Note that if $T$ is a partial tilting module, then an Ext-projective complement $T^{\prime}$ in $T^{\perp}$ correspond to the Bongartz complement as $T^{\perp}=\left(T \amalg T^{\prime}\right)^{\perp}$. As a further application of our characterizations of tilting and cotilting modules we obtained the following. The different proofs are given in [2, Theorem 2.1] and [47, Theorem 6.2].

Theorem 4.2. $\quad$ (a) Let $T$ be a partial tilting module. Then $T$ has a complement which is Ext-projective in $T^{\perp}$ if and only if $T^{\perp}$ is closed under coproducts.

(b) Let $T$ be a partial cotilting module. Then $T$ has a complement which is Ext-injective in ${ }^{\perp} T$ if and only if ${ }^{\perp} T$ is closed under products and each $\Lambda$-module has a special right ${ }^{\perp} T$-approximation.

Here we see that the statements of the results for tilting and cotilting are not dual. The explanation for this is the following. By [35, Theorem 10] every $\Lambda$-module has a special left $M^{\perp}$-approximation for any $\Lambda$-module $M$, while it is unknown if every $\Lambda$-module has a special right ${ }^{\perp} M$-approximation for any $\Lambda$-module $M$. However, "duality" is restored if we assume that $T$ is pure-injective, since by [34, Corollary 10] every $\Lambda$-module has a minimal right ${ }^{\perp} M$-approximation whenever $M$ is pureinjective. In fact, we have the following, where the equivalence of (a) and (c) can be found in [2, p. 93].

Proposition 4.3. [47, Corollary 6.3] Let $T$ be a pure-injective partial cotilting module. The following are equivalent.

(a) $T$ admits a complement which is Ext-injective in ${ }^{\perp} T$,

(b) $T$ admits a pure-injective complement which is Ext-injective in ${ }^{\perp} T$,

(c) ${ }^{\perp} T$ is closed under products.

Let us proceed by addressing the first question for cotilting modules: (1) Does the Bongartz construction of a complement generalize to cotilting modules of injective dimension at most one in $\operatorname{Mod} \Lambda$ ? In [11] any cotilting module of injective dimension at most one is shown to be pure-injective. Hence a necessary assumption for being 
able to answer question (1) affirmatively is to start with a pure-injective partial cotilting module of injective dimension at most one. This is also shown to be sufficient in [17, Corollary 1.12].

Proposition 4.4. Suppose that $T$ is a pure-injective partial cotilting module of injective dimension at most one. Then there exists a pure-injective module $T^{\prime}$ such that $T \amalg T^{\prime}$ is a pure-injective cotilting module.

Proof. Let $M$ be a pure-injective module of injective dimension at most one. It is shown in [17, Corollary 1.10] that ${ }^{\perp} M$ is closed under products. The claim then follows immediately from the above result.

It follows from [17, Lemma 1.11] that the module $T^{\prime}$ found in the above proposition corresponds to the Bongartz construction.

For partial tilting modules the answer of question (1) is not so complete. Recall that a class of modules $\mathcal{X}$ is said to be of finite (countable) type if $\mathcal{X}=\mathcal{S}^{\perp}$ for some subset $\mathcal{S}$ of finitely presented (countably generated) modules in $\mathcal{P}^{n+1}(\operatorname{Mod} \Lambda)$ for some $n<\infty$. A module $M$ is of finite or countable type if the subcategory $M^{\perp}$ is of finite or countable type, respectively. In [13] all tilting modules of projective dimension at most one are shown to be of countable type, and it is open if all tilting modules of projective dimension at most one are of finite type. If $M$ is a module of finite type, then $M^{\perp}$ is closed under products, filtered colimits and pure submodules, in particular closed under coproducts. As a consequence of this and Theorem 4.2 (a) we obtain the following.

Proposition 4.5. Suppose that $T$ is a partial tilting module of finite type (and of projective dimension at most one). Then there exists a module $T^{\prime}$ such that $T \amalg T^{\prime}$ is a tilting module of finite type (and of projective dimension at most one).

We end this section by answering the second question: Do any partial tilting or cotilting module $T$ in $\bmod \Lambda$ have a complement when considered as a partial tilting or cotilting module in $\operatorname{Mod} \Lambda$ ? Since $T^{\perp}$ is closed under coproducts and ${ }^{\perp} T$ is closed under products (actually definable) when $T$ is finitely presented, the following result is an immediate consequence of Theorem 4.2 (see [2, Corollary 2.2] and [47, Corollary 6.4]).

Proposition 4.6. Let $T$ be a finitely presented $\Lambda$-module.

(a) If $T$ is a partial tilting module, then $T$ has a complement which is Extprojective in $T^{\perp}$.

(b) If $T$ is a partial cotilting module, then $T$ has a complement which is pureinjective and Ext-injective in ${ }^{\perp} T$.

\section{Classification of All Cotilting modules}

For an artin algebra $\Lambda$ of finite representation type all modules in $\operatorname{Mod} \Lambda$ is isomorphic to a direct sum of indecomposable finitely presented modules (see [7, 56]). Therefore every tilting or cotilting module is equivalent to a finitely presented tilting or cotilting module, hence there is only a finite number of them.

For algebras of infinite representation type a complete classification of all cotilting modules seems only to be known for tame hereditary algebras $([17,18])$. The aim of this section is to give this classification. For information on cotilting modules over concealed canonical algebras see [52, Section 10]. 
Assume throughout that $\Lambda$ is a finite dimensional tame hereditary algebra. We start by reviewing some facts about these algebras (see [55]). The category of finitely presented regular modules is denoted by $\mathcal{R}$, which are all the modules occurring in the tubes of the Auslander-Reiten quiver of $\Lambda$. This category is an abelian category, and $\mathbb{P}$ denotes the set of isomorphism classes of all simple objects in $\mathcal{R}$. Two elements $S$ and $S^{\prime}$ in $\mathbb{P}$ are said to be equivalent if $\operatorname{Ext}_{\Lambda}^{1}\left(S, S^{\prime}\right) \neq(0)$ or $\operatorname{Ext}_{\Lambda}^{1}\left(S^{\prime}, S\right) \neq(0)$. Take the transitive closure of this relation in $\mathbb{P}$, and let $[S]$ denote the equivalence class of $S$. For each $S$ in $\mathbb{P}$ there are unique indecomposable objects $S_{n}$ and $S_{-n}$ of length $n$ in $\mathcal{R}$ such that $\operatorname{Hom}_{\Lambda}\left(S, S_{n}\right) \neq(0)$ and $\operatorname{Hom}_{\Lambda}\left(S_{-n}, S\right) \neq(0)$. Moreover there are chains of monomorphisms $S=S_{1} \rightarrow$ $S_{2} \rightarrow \cdots$ and chains of epimorphisms $\cdots \rightarrow S_{-2} \rightarrow S_{-1}=S$ for each $S$ in $\mathbb{P}$. The colimit $\lim S_{n}=S_{\infty}$ is the corresponding Prüfer module, and the inverse limit $\lim _{\longleftarrow} S_{-n}=\overleftrightarrow{S_{-\infty}}$ is the adic module. Finally there is a unique generic module $G$, that is, $G$ is indecomposable of infinite length and has finite length over $\operatorname{End}_{\Lambda}(G)$.

Given a module $M$ in $\operatorname{Mod} \Lambda$, denote by indec $M$ the set of all the isoclasses of indecomposable direct summands of $M$. If $M$ is pure-injective, then there is a unique family $\left\{M_{i}\right\}_{i \in I}$ of modules in indec $M$ such that $M$ is the pure-injective envelope of $\amalg_{i \in I} M_{i}$. With these preliminaries we can give the classification of all cotilting modules over $\Lambda$. Recall that by [11] all cotilting modules of injective dimension at most one are pure-injective, so that all cotilting modules over $\Lambda$ are pure-injective.

Theorem 5.1. [17, Theorem 3.9] Let $\Lambda$ be a tame hereditary algebra, and let $T$ be a pure-injective $\Lambda$-module.

(a) Suppose that all indecomposable direct summands of $T$ are finitely presented. Then $T$ is a cotilting module if and only if card(indec $T$ ) equals the number of non-isomorphic simple $\Lambda$-modules and $\operatorname{Ext}_{\Lambda}^{1}\left(T^{\prime}, T^{\prime \prime}\right)=(0)$ for all $T^{\prime}$ and $T^{\prime \prime}$ in indec $T$.

(b) Suppose there is an indecomposable direct summand of $T$ which is not finitely presented. Then $T$ is a cotilting module if and only if the following holds:

(i) Each $M$ in indec $T$ is either generic or of the form $S_{n}$ for some $S$ in $\mathbb{P}$ and some $n$ in $\mathbb{N} \cup\{-\infty, \infty\}$.

(ii) For each $S$ in $\mathbb{P}$, the set $I_{S}$ consisting of all the non-isomorphic modules $M$ in indec $T$ with $M \simeq S_{n}^{\prime}$ for some $n$ in $\mathbb{N} \cup\{-\infty, \infty\}$ and some $S^{\prime}$ in $[S]$ satisfies card $I_{S}=\operatorname{card}[S]$ and $\operatorname{Ext}_{\Lambda}^{1}\left(T^{\prime}, T^{\prime \prime}\right)=(0)$ for all $T^{\prime}$ and $T^{\prime \prime}$ in $I_{S}$.

(c) Two cotilting modules $T_{1}$ and $T_{2}$ are equivalent if and only if $\operatorname{indec}\left(T_{1} \amalg G\right)=$ $\operatorname{indec}\left(T_{2} \amalg G\right)$.

The cotilting modules occurring in part (b) are given by for every tube choosing the rank of the tube indecomposable non-isomorphic modules totally from the tube, its Prüfer module or its adic module. For a tube of rank one there are only two choices, the Prüfer or the adic module. A complete characterization of the different possible choices in a tube is given in [18].

\section{REFERENCES}

[1] Aldrich, S. T., Enochs, E. E., Jenda, O. M. G., Oyonarte, L., Envelopes and covers by modules of finite injective and projective dimension, J. Algebra 242 (2001), 447-459. 
[2] Angeleri-Hügel, L., Coelho, F. U., Infinitely generated complements to partial tilting modules, Math. Proc. Cambridge Philos. Soc. 132 (2002), no. 1, 89-96.

[3] Angeleri-Hügel, L., Coelho, F. U., Infinitely generated tilting modules of finite projective dimension, Forum Math. 13 (2001), no. 2, 239-250.

[4] Angeleri-Hügel, L., Tonolo, A., Trlifaj, J., Tilting preenvelopes and cotilting precovers, Algebras and Representation Theory, 4 (2001), 155-170.

[5] Angeleri-Hügel, L., Trlifaj, J., Tilting theory and the finitistic dimension conjectures, Trans. Amer. Math. Soc. 354 (2002), no. 11, 4345-4358.

[6] Assem, I., Tilting theory - an introduction, Topics in Algebra, Banach Center Publications 26 PWN, Warsaw (1990), 127-180.

[7] Auslander, M., Representation theory of artin algebras II, Comm. Algebra 1 (1974), 269-310. 1976.

[8] Auslander, M., Platzeck, M. I., Reiten, I., Coxeter functors without diagrams, Trans. Amer. Math. Soc. 250 (1979), 1-12.

[9] Auslander, M., Reiten, I., Applications of contravariantly finite subcategories, Adv. Math. 86 (1991) no. 1, 111-152.

[10] Auslander, M., Smalø, S. O., Almost split sequences in subcategories, J. Algebra 69 (1981), no. 2, 426-454. Addendum: J. Algebra 71 (1981), no. 2, 592-594.

[11] Bazzoni, S., Cotilting modules are pure-injective, Proc. Amer. Math. Soc. 131 (2003), no. 12, 3665-3672.

[12] Bazzoni, S., n-cotilting modules and pure-injectivity, Bull. London Math. Soc., to appear.

[13] Bazzoni, S., Eklof, P., Trlifaj, J., Tilting cotorsion pairs, preprint.

[14] Bernstein, I., Gelfand, I. M., Ponomarev, V. A., Coxeter functors and Gabriel's theorem, Usp. Mat. Nauk 28 (1973), 19-23.

[15] Bongartz, K., Tilted algebras, Representations of algebras (Puebla, 1980), Lecture Notes in Math. 904, Springer, Berlin-New York (1981), 26-38.

[16] Brenner, S., Butler, M. C. R., Generalizations of the Bernstein-Gelfand-Ponomarev reflection functors, Lecture Notes in Math. 832, Springer-Verlag, Berlin, 1980, 103-169.

[17] Buan, A. B., Krause, H., Cotilting modules over tame hereditary algebras, Pacific J. Math. 211 (2003), no. 1, 41-59.

[18] Buan, A. B., Krause, H., Tilting and cotilting for quivers of type $\widetilde{A}_{n}$, J. Pure Applied algebra, to appear.

[19] Buan, A. B., Krause, H., Solberg, Ø., On the lattice of cotilting modules, AMA Algebra Montp. Announc. 2002, Paper 2, 6 pp.

[20] Buan, A. B., Solberg, Ø., Limits of pure-injective cotilting modules, J. Algebra and Representation Theory, to appear.

[21] Buan, A. B., Solberg, Ø., Relative cotilting theory and almost complete cotilting modules, Algebras and modules II, CMS Conf. Proc. 24 (1998), 77-92.

[22] Colby, R. R., A generalization of Morita duality and the tilting theorem, Comm. Algebra 17 (1989), 1709-1722.

[23] Colby, R. R., A cotilting theorem for rings, Methods in Module Theory, M. Dekker, New York, 1993, pp. 33-37.

[24] Colby, R. R., Fuller, K. R., Tilting and torsion theory counter equivalences, Comm. Algebra 23 (1995), 4833-4849.

[25] Colby, R. R., Fuller, K. R., Tilting and serially tilted algebras, Comm. Algebra 23 (1995), $1585-1616$.

[26] Colpi, R., Cotilting bimodules and their dualities, Proc. Euroconf. Murcia '98, LNPAM 210, M. Dekker, New York 2000, 81-93.

[27] Colpi, R., D'Este, G., Tonolo, A., Quasi-tilting modules and counter equivalences, J. Algebra 191 (1997), 461-494.

[28] Colpi, R., Fuller, K. R., Cotilting modules and bimodules, Pacific J. Math. 192 (2) (2000), 275-291.

[29] Copli, R. R., Tonolo, A., Trlifaj, J., Partial cotilting modules and the lattice induced by them, Comm. Algebra 25 (1997), 3225-3237.

[30] Copli, R. R., Trlifaj, J., Tilting modules and tilting torsion theories, J. Algebra 178 (1995), 614-634.

[31] Crawley-Boevey, W., Locally finitely presented additive categories, Comm. Algebra 22 (1994), 1644-1674. 
[32] Dräxler, P., Happel, D., A proof of the generalized Nakayama conjecture for algebras with $J^{2 l+l}=0$ and $A / J^{l}$ representation finite, J. Pure Appl. Algebra 78 (1992), no. 2, 161-164.

[33] Erdmann, K., Holm, T., Iyama, O., Schröer, J., Radical embeddings and representation dimension, Adv. Math., to appear.

[34] Eklof, P., Trlifaj, J., Covers induced by Ext, J. Algebra 231 (2000), 640-651.

[35] Eklof, P., Trlifaj, J., How to make Ext vanish, Bull. London Math. Soc., 33 (2001), 41-51.

[36] Green, E. L., Kirkman, E.., Kuzmanovich, J., Finitistic dimensions of finite dimensional monomial algebras, J. Algebra 136 (1991), no. 1, 37-50.

[37] Green, E. L., Huisgen-Zimmermann, B., Finitistic dimension of artinian rings with vanishing radical cube, Math. Z. 206 (1991), no. 4, 505-526.

[38] Happel, D., Triangulated categories in the representation theory of finite-dimensional algebras, London Math. Soc. Lecture Notes Series, 119. Cambridge Univ. Press (1988).

[39] Happel, D., Ringel, C. M., Tilted algebras, Trans. Amer. Math. Soc. 274 (1982), 399-443.

[40] Happel, D., Unger, L., Complements and the Generalized Nakayama conjecture, Algebras and modules II, CMS Conf. Proc. 24 (1998), 293-310.

[41] Huisgen-Zimmermann, B., Homological domino effects and the first finitistic dimension conjecture, Invent. Math. 108 (1992) 369-383.

[42] Huisgen-Zimmermann, B., Smalø, S. O., A homological bridge between finite and infinite dimensional representations, Algebras and Representation Theory 1 (1998), 169-188.

[43] Igusa, K., Todorov, G., On the finitistic global dimension conjecture for artin algebras, preprint.

[44] Igusa, K., Zacharia, D., Syzygy pairs in a monomial algebra, Proc. Amer. Math. Soc. 108 (1990), no. 3, 601-604.

[45] Jensen, C. U., Lenzing, H., Model-theoretic algebra with particular emphasis on fields, rings, modules, Algebra, Logic and Applications, 2. Gordon and Breach Science Publishers, New York, 1989, xiv+443 pp.

[46] Krause, H., Solberg, Ø., Application of cotorsion pairs, J. London Math. Soc. (2) 68 (2003), no. 3, 631-650.

[47] Krause, H., Solberg, Ø., Filtering modules of finite projective dimension, Forum Math. 15 (2003), no. 3, 377-393.

[48] Mantese, F., PhD-thesis, University of Padova, 2003.

[49] Menini, C., Orsatti, A., Representable equivalences between categories of modules and applications, Rend. Sem. Math. Univ. Padova 82 (1989), 203-231.

[50] Miyashita, Y., Tilting modules of finite projective dimension, Math. Z. 193 (1986), 113-146.

[51] Reiten, I., Tilting and homologically finite subcategories with application to quasi-hereditary algebras, this volume.

[52] Reiten, I., Ringel, C. M., Infinite dimensional represetations of canonical algebras, preprint.

[53] Rickard, J., Schofield, A., Cocovers and tilting modules, Math. Proc. Cambridge Philos. Soc. 106 (1989), no. $1,1-5$.

[54] Riedtmann, C., Schofield, A., On a simplicial complex associated with tilting modules, Comment. Math. Helv. 66 (1991), 70-78.

[55] Ringel, C. M., Infinite-dimensional representations of finite-dimensional hereditary algebras, Symposia Mathematica, Vol. XXIII (Conf. Abelian groups and their Relationship to the Theory of Modules, INDAM, Rome, 1977), Academic Press, London-New York *1979), 321412.

[56] Ringel, C. M., Tachikawa, H., QF-3 rings, J. Reine Angew. Math. 272 (1974) 49-72.

[57] Smalø, S. O., Torsion theories and tilting modules, Bull. London Math. Soc. 16 (1984), 518522.

[58] Tonolo, A., Tilting modules of finite projective dimension: sequentially static and costatic modules, J. Algebra Appl. 1 (2002), 295-305.

[59] Trlifaj, J., Infinite dimensional titling modules and cotorsion pairs, this volume.

[60] Unger, L., Shellability of simplicial complexes arising in representation theory, Adv. Math. 144 (1999), no. 2, 211-246.

[61] Unger, L., The collection of tilting modules and its combinatorics, this volume.

[62] Wakamatsu, T., On modules with trivial self-extensions, J. Algebra 114 (1988) 106-114.

Øyvind Solberg, Institutt for matematiske fag, NTNU, N-7491 Trondheim, Norway

E-mail address: oyvinso@math.ntnu.no 